\title{
Biochemical Effect of Beta-blockers on Thyroid Function and Lipid Metabolism in Experimentally Induced Hyperlipidemia in Rats
}

O.M.Abdel-Hamied ${ }^{1}$,A.A.Nafeaa ${ }^{2}$ and H.A.Ghoneimy ${ }^{3}$

${ }^{1}$ Clinical Biochemistry Dept., Faculty of Veterinary Medicine, Benha Univ., Benha, Egypt

${ }^{2}$ Physiology Dept., Faculty of Veterinary Medicine, Benha Univ., Benha, Egypt

${ }^{3}$ Faculty of Science, Zagazig Univ.,El-Sharkia, Egypt

E-Mail: omnia.hassan@fvtm.bu.edu.eg

\begin{abstract}
The present study was employed to investigate the biochemical effects of Propranolol or Atenolol adminstration as non-selective or selective beta-blockers, respectivly on thyroid functions and lipid metabolism. Forty male albino rats were assigned into two groups (the first group contained 10 rats fed on normal diet while the second group contained 30 rats used for induction of hyperlipidemia. Then the second group was divided into 3 sub groups (each of 10 rats). Sub group 1: act as positive control hyperlpidemic rats, sub group 2: adminstrated propranolol orally while sub group 3: adminstrated atenolol orally. Then blood samples were collected after 3 and 5 weeks from the drugs adminstration. Serum was collected for estimation of Triiodothyronine (T3), Tetraiodothyronine (T4), Thyroid Stimulating Hormone (TSH), Total Cholesterol, High Density Lipoprotein Cholesterol (HDL-C), Low Density Lipoprotein-Cholesterol (LDL-C), Very Low Density Lipoprotein-Cholesterol (VLDL), Triacyclglycerols (TAG), Phospholipids, Leptin, Lipoprotein lipase (LPL), Lactate dehydrogenase (LDH), Creatine Kinase (CK) and Creatine Kinase-MP (CK-MP) as isoenzyme, Calcium and phosphorous. The obtained results revealed that, propranolol has more biochemical effect in significant decreasing (T3) than atenolol with non-significant decrease in serum (T4), both of propranolol and atenolol has no effects on TSH, cholesterol. Both of propranolol and atenolol has the same effects in significant decreasing HDL and lipase activity. Propranolol has more biochemical effect in significant increasing TAG, VLDL, phospholipids, LDH than Atenolol. Both of propranolol and atenolol has the same effect in significant increasing serum leptin.
\end{abstract}

Keywords: Atenolol, Cardiac markers, Hyperlipidemia, Lipid profile, Propranolol, Thyroid hormones.

\section{Introduction}

Nowadays, the blood pressure plays an effective role in our medical life, Hypertension or hypotension causes coma which continuously cause death, one from four adults suffer from high blood pressure, putting them at serious risk of heart failure, stroke, blindness, and premature death [9]. The most important cause of blood pressure disturbance is hyperlipidemia which well known to affect the development of atherosclerosis; accumulation of lipids in the arterial wall plays a crucial role in the genesis of atherosclerosis by impairing endothelial function [18]. Doctors stile prescribe beta-blockers non-cardioselective as propranolol or cardioselective as atenolol for patients with tachycardia (rapid heart rates), to help patients with angina by lowering the amount of oxygen that heart muscles require. Several studies showed the mechanism of antihypertensive effects of beta adrenoceptore antagonist depending on the oxygen domain and up take by heart tissues [23].

The most common underlying form of thyroid disease associated with thyrotoxic periodic paralysis (TPP) is Graves' disease, a syndrome due to an autoimmune reaction that leads to overproduction of thyroid hormones. [16] showed the mechanism of TPP and the role of beta-blockers in treatment of the muscle weakness and increased risk of irregular heart beat in TPP. Beta-blockers are described also for patients with tachycardia. [32] reported that during the 1970 s to the mid1990s, numerous studies were performed with results advocating the use of beta-blockers, in particular propranolol, alone or in combination with iodine in the perioperative treatment and stabilization of the thyrotoxic patient. This regime can safely provide rapid control of the peripheral manifestations of hyperthyroidism, producing a clinically euthyroid patient in a relatively short period.

\section{Materials and methods}

Forty white male albino rats of 8-10 weeks old and 150-180 g. body weight were housed in separated metal cages and kept in constant environmental conditions throughout the period of the experiment. Animals were fed on a constant ration and water was supplied ad libitum. Rats were assigned into two groups (the first group contained 10 rats which were fed on a normal diet while the second group contained 30 rats used for induction of hyperlipidemia.

\subsection{Induction of hyperlipidemia}

Hyperlipidemia was induced by feeding 30 rats on lipid rich diet (93 g. of normal ration $+1 \mathrm{gm}$. Cholesterol with +1 g. Cholic powder $+5 \mathrm{~g}$. butter) for 60 days. After 60 days, estimation of serum triacylglycerol and cholesterol showed hyperlipidemia. 
2.2Preparation of propranolol and atenolol doses

The dose was chosen to be within the therapeutic range levels reported in the pamphlet that indicated that, the limited dose for human equals $360 \mathrm{mg} / \mathrm{kg} /$ day, but for rats it equals; $360 \mathrm{x}$ $0.018=6.48 \mathrm{mg} / \mathrm{kg} /$ day. The medium weight of rats was $190 \mathrm{~g}$. so, the required dose for each one was; $(190 \times 6.48) / 1000=1.23 \mathrm{mg} /$ day. One tablet of Inderal $40 \mathrm{mg}$ was dissolved in $10 \mathrm{ml}$ of distilled water to give a solution each $1 \mathrm{ml}$ contains $4 \mathrm{mg}$ so the dose of each rat was $(1.23 / 4=0.308 \mathrm{ml})$ once daily using stomach tube for administration. One tablet of Tenormin $100 \mathrm{mg}$ obtained from Cairo company for medicine industries was dissolved in $10 \mathrm{ml}$ of distilled water to give a solution each $1 \mathrm{ml}$ contains $10 \mathrm{mg}$ so the dose of each rat was $(1.23 / 10$ $=0.123 \mathrm{ml}$ ) once daily using stomach tube for administration [21].

\subsection{Experimental design}

Animals were assigned into two groups,

Group I (normal rats): 10 rats were fed on a normal ration.

Group II (Hyperlipidemic group): 30 rats were fed on lipid rich ration for 60 day after examining the lipid profile this group was allocated into 3 subgroups; each one contained 10 rats.

A. Control hyperlipidemic subgroup (1): rats act as control positive.

B. Hyperlipidemic propranolol subgroup (2); rats were administrated nonselective beta-blocker (0.308 ml propranolol solution once daily using a stomach tube).

C. Hyperlipidemic atenolol subgroup (3): rats were administrated atenolol as selective betablocker $(0.123 \mathrm{ml}$ atenolol solution once daily using a stomach tube).

\subsection{Sampling}

Blood samples were collected from the retroorbital venous plexus after 3 and 5 weeks from the drugs administration. Every sample was collected in dry and clean tube without anticoagulant then centrifuged at $3000 \mathrm{rpm}$ for 10 minutes to separate the serum. The clear serum samples were aspirated carefully by Pasteur pipettes and transferred into dry, clean and sterile labeled tubes for processing the following biochemical analysis: Serum total (T3) was determined according to the method described by [35]. Serum total (T4) was determined according to the method described by [36] and Thyroid Stimulating Hormone (TSH) was determined according to the method described by [29] using RIA kits, Total cholesterol was determined enzymatically according to [34], High density lipoprotein cholesterol (HDL-C) was determined by enzymatic method according to [17], Low density lipoprotein cholesterol (LDL-C) can be calculated using the formula described by [7], Very low density lipoproteins (VLDL) can be calculated using the formula described by [2], Triacyclglycerols (TAG) was determined according to [8], Lipoprotein lipase (LPL) was determined according to [15], Phospholipids were determined according to [22], Leptin was estimated by enzyme linked immunosorbent assay (ELIZA) kits, Lactate dehydrogenase (LDH) was determined according to [27], Creatine kinase (CK) was determined according to the method described by [40], Creatine kinase- MP (CK-MP) was determined according to the method of [33], serum calcium level was determined according to [10], serum inorganic phosphorous level according to the method described by [39].

\subsection{Statistical analysis}

The Statistical analysis was carried out using ANOVA with two factors under significance level of 0.05 for the whole results using SPSS ver. 19 . Data were treated as complete randomization design according to [30]. Multiple comparisons were carried out applying LSD.

\section{Results}

The obtained data in table 1 and 2 revealed that, administration of propranolol or atenolol to hyperlipidemic rats resulted in a significant decrease in serum (T3), serum (HDL-C), serum lipase concentration after 3 and 5 weeks in comparison with hyperlipidemic control group. Administration of atenolol in hyperlipidemic rats resulted in a significant decreasing in serum calcium level, while administration of propranolol in hyperlipidemic rats resulted in a non-significant decreasing in serum calcium level.

Hyperlipidemic propranolol rats showed a significant decrease in serum (CK-MB) activity after 3 and 5 weeks while hyperlipidemic atenolol rats showed a non-significant decrease in serum (CK-MB) concentration after 3 weeks in comparison with hyperlipidemic control group, but after 5 weeks showed a significant decrease when compared with hyperlipidemic control group.

Hyperlipidemic propranolol and hyperlipidemic atenolol groups showed a nonsignificant effect in serum (T4), (TSH), cholesterol, (LDL-C) after 3 and 5 weeks from the start of administration in comparison to hyperlipidemic control group.

Administration of propranolol or atenolol in hyperlipidemic rats showed a significant increase in serum (VLDL), serum (TAG), serum phospholipids, serum leptin and serum inorganic phosphorous level when compared with hyperlipidemic control group during all the periods of experiment. Hyperlipidemic propranolol group showed a significant increase in serum (LDH) concentration when compared with hyperlipidemic atenolol group during all the periods of experiment. 


\section{Discussion}

The obtained results showed in table 1 and 2 revealed that, propranolol and atenolol produced significant decreases in serum (T3) concentration in hyperlipidemic rats with euthyroid state. At the end of experiment $\left(5^{\text {th }}\right.$ week) propranolol significantly (at $p<0.05$ ) decreased the mean serum (T3) concentration in the third group by $12.5 \%$, while atenolol significantly (at $p<0.05$ ) decreased the mean serum (T3) concentration in the fourth group by $8.8 \%$, whereas, we found that the administration of hyperlipidemic rats with propranolol or atenolol resulted in non-significant (at $p<0.05$ ) decrease in serum (T4) concentration after 3 and 5 weeks of administration also, our results referred to nonsignificant change in serum (TSH) concentration in this tow hyperlipidemic rats groups this was statistically non-significant (at $p<0.05$ ). These results were in agreement with [19] who reported that, propranolol and atenolol drugs do not act like anti-thyroid drugs by reducing thyroid hormone secretion. However, it has been documented by the results that the non-selective $\beta$ blocker propranolol can decrease the level of triiodothyronine (T3) without affecting thyroxine (T4) level. This effect appears to be through the inhibition of 5'monodeiodinase, a microsomal enzyme responsible for the peripheral conversion of (T4) to (T3). [6] Proved that, propranolol is the most commonly used beta-blocker to treat thyrotoxicosis where it produces a rise in rT3 (resistant of triiodothyronine) and has been the mainstay beta-blocker therapy to prepare thyrotoxic patients for surgery. [24] indicated that atenolol is as effective as propranolol in reducing serum total (T3) concentration due to increasing the resistant (T3) in thyrotoxic rats.

Administration of propranolol or atenolol produced undesirable effect on lipid profile, the obtained results were in agreement with [1] who studied the effects atenolol on hypertensive patients and found that there is no significant change in total cholesterol (TC) and HDL-c is significantly lowered in patients treated with atenolol in comparison with hyperlipidemic control group, the supposed explanation for this effect was introduced by [11] who stated that the decrease in serum HDLc might be accounted to be due to the inhibitory effect of $\beta$ - blockers on lipoprotein lipase. The level of total lipids was significantly higher in Atenolol using rats due to increasing triglycerides and decreasing the level of lipase enzyme under the effect of beta-blockers. This result is in agreement with the result obtained by other authors who found that atenolol causes a significant increase in total cholesterol / HDL-c ratio ( i.e. AI) [3], that might be explained by the significant change in serum HDL-c level by atenolol in the present study. The comparison of serum triglycerides level in patient's group using atenolol with the control group shows that there is a significant increase in serum triglycerides level in those using atenolol. This result is in agreement with that result of other authors where they found that atenolol causes hypertriglyceridemia [1]. The obtained results were in disagreement with [4] who showed that beta blockers did not adversely influence total cholesterol, HDL cholesterol, or LDL cholesterol, beta blockers had no undesirable effects on total cholesterol, HDL cholesterol, LDL cholesterol and triglycerides in 42 patients studied over 10 months, The study with a follow-up of three years that bisoprolol did not adversely affect HDL cholesterol or triglycerides, while propranolol and atenolol both deteriorated these lipid parameters.

The obtained data in table $(1,2)$ showed that a significant decrease in lipase enzyme of rats which are treated with atenolol all over the experiment and this agreement with [14] recorded that, In rats fed a normal lab chow, propranolol decreased heart lipoprotein lipase activity. This effect was not statistically significant when compared with no treated controls; there were no significant effects on adipose tissue lipoprotein lipase activity or hepatic lipase activity. In rats fed a cholesterol-enriched diet there were similar but smaller effects on heart lipoprotein lipase activity. Our results are in disagreement with [28] where they found that the long administration of atenolol with low dose decreasing the serum lipase on contrary short administration of atenolol with high dose gives high level of lipase, but we found that long administration of atenolol decreasing the lipase level with reference to hyperlipidemic state of our research objectives.

The effect of beta blockers on phospholipids was studied by [38] who reported that, the beta blocking ocular hypotensive drugs, propranolol and atenolol, produced varying effects on 32P incorporation into phospholipids of iris and ciliary process. Propranolol stimulated 32Pi-labelling into phosphatidylinositol 4', 5' bisphosphate (PIP2), phosphatidylinositol 4' phosphate (PIP), PI and PA., whereas atenolol, had no significant effect on 32Pilabelling of phospholipids.

The obtained data revealed a significant increase in serum liptin concentration of rats fed on 
high fat diet all over the period of experiment, compared to the normal rats that received basal diet. Our results are in agreement with [20] Stated that, leptin is at least in part controlled neuronally because anesthesia decreased serum leptin and blocked its response to LPS (Lipopolysaccharide). The findings that propranolol increased serum leptin concentrations suggest that leptin release is inhibited by the sympathetic nervous system. Propranolol produced a significant increase in serum leptin but had no effect on the response to LPS. [37] indicated the increase of the leptin receptor level in the hypothalamus by propranolol, indicating an enhanced leptin signaling in the brain. Thus, propranolol controls the leptin signaling in two distinct directions.

There is a significant decrease in serum creatine kinase concentration in hyperlipidemic propranolol and atenolol groups, these results are in agreement with [26] who reported that the beneficial effect of beta-blockers did not seem to be mediated by the prevention of CK-MB elevation, because a statistically significant decrease benefit was observed only in patients with normal CK-MB values. Beta-blockade decreases oxygen demand by preventing increases in heart rate and blood pressure. Our results were in contrast with [13] recorded that, increased serum levels of CK-MB in hypertensive patients taking atenolol are directly proportional to the duration of the atenolol usage. Long exposure of cardiac muscle to atenolol leads to escape of CK-MB to circulation, this may be due to cardiac muscle which becomes fatigue with prolonged exposure to atenolol causing it unable to contract efficiently and ending with failure.

The results are in agreement with [25] investigated that group treated with atenolol showed non-significant increase in serum LDH level compared to normal group. The effect of atenolol could be due to its $\beta$-blocking effect, which prevents membrane damage by reducing the cardiac workload. [12]. Investigated that serum LDH activities were found to be increased in diabetic rats, possibly due to myocardial dysfunction because it has been previously reported that serum $\mathrm{LDH}$ activities were found to be increased in cardiomyopathy. Serum LDH levels were also reported to increase in diabetic patients, and may serve as a marker for cardiovascular risk and cardiac muscular damage. In the present study, there was a significant decrease in serum LDH levels observed with treatment of atenolol, carvedilol and nebivolol indicating good cardioprotection.

For showing the explanation of beta-blockers effect on serum calcium and phosphorous levels there was a study for [31] recorded that concentration of sodium, potassium, calcium and magnesium was increased in serum after atenolol treatment. Occur by suppression in renin release. It is suggested that renin release is suppressed by small doses of all beta blockers. The fall in blood pressure in response to beta blocker therapy may be related directly to changes in plasma renin activity and thus increased serum potassium. The decreased tissue sodium also decreases the calcium influx through Na-Ca-exchange mechanism and thus caused the decreased intracellular calcium content. The decreased intracellular calcium results in a vasorelaxant action and lowering the blood pressure. The obtained results did not agree with [5] stated that significant elevation in serum in organic phosphate occurred during nadolol treatment and a similar elevation has previously been reported during treatment of hyperthyroid patients with propranolol and metoprolol, this means that propranolol, practolol or metoprolol induce the change in serum calcium level in thyrotoxic patients .

\section{Conclusion}

From these results we concluded that, these drugs are recommended for hypertensive patients with thyrotoxicosis or cardiovascular diseases but they have some undesirable effects on the metabolism of lipids. 


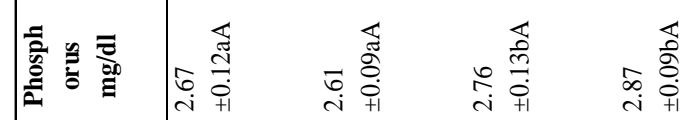

旁望

है

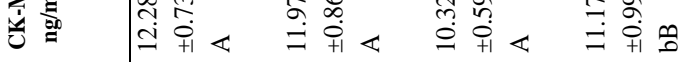

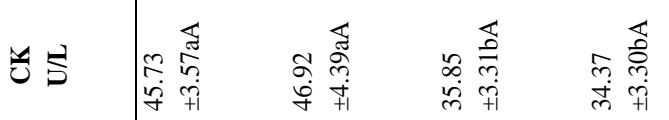

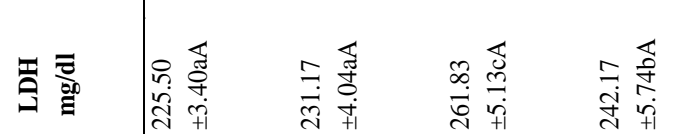

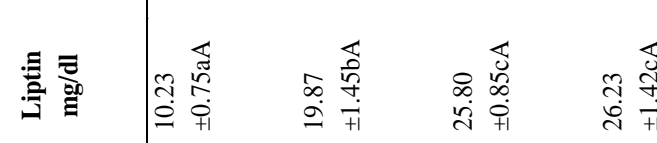

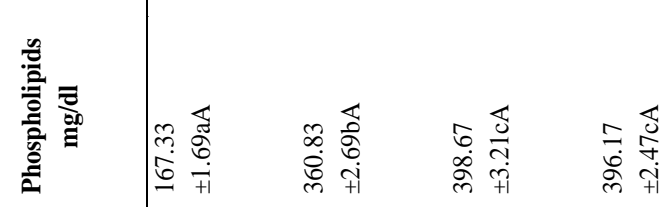

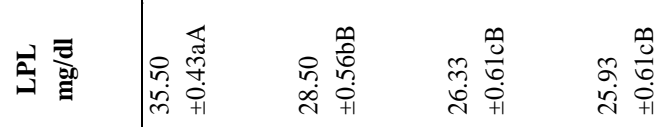

$\frac{\dot{n}}{\mathbb{8}}$

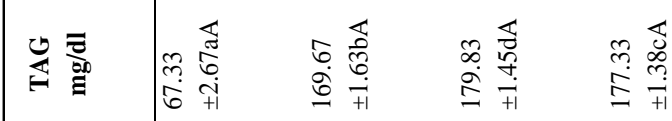

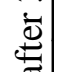

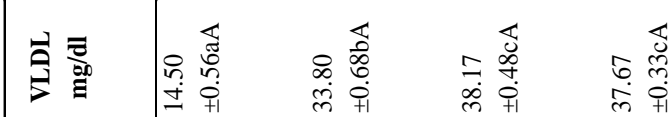

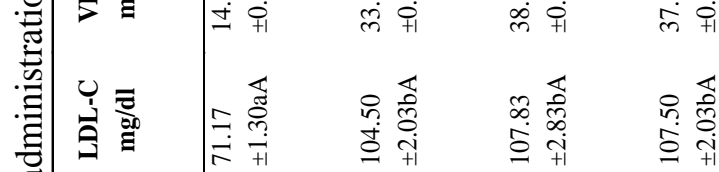

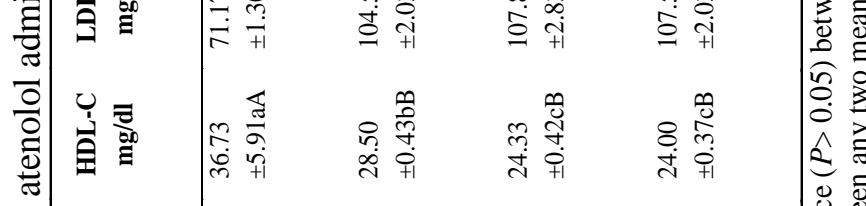

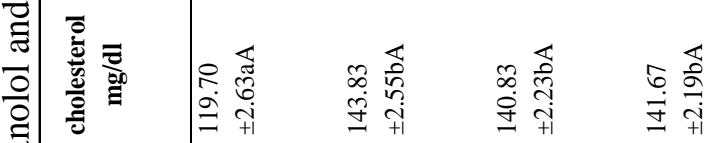

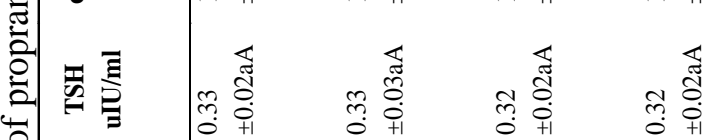

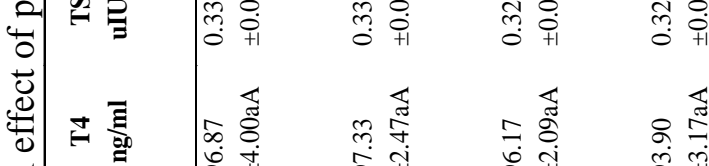

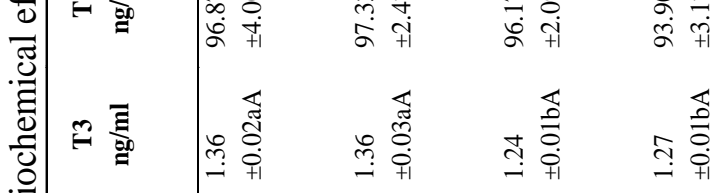

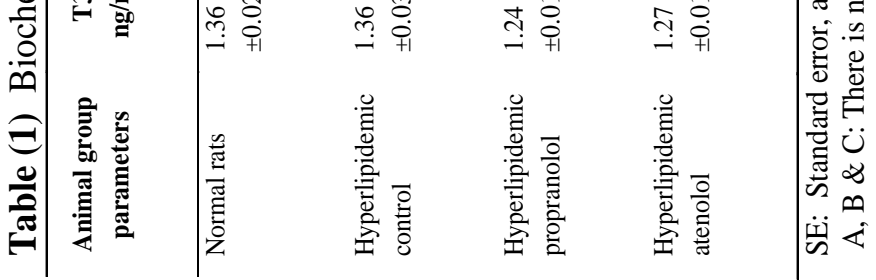

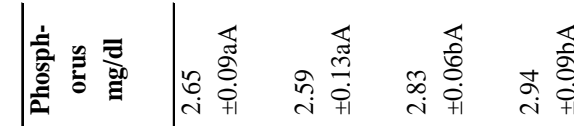

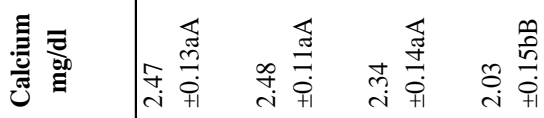

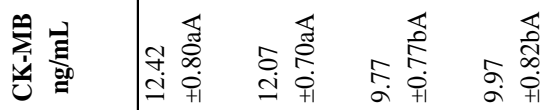

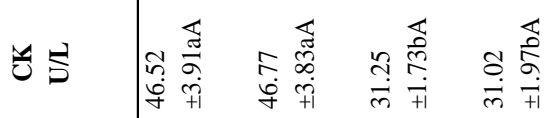

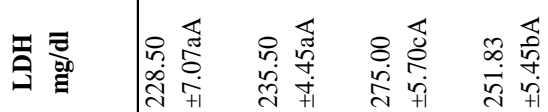

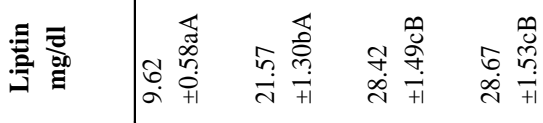

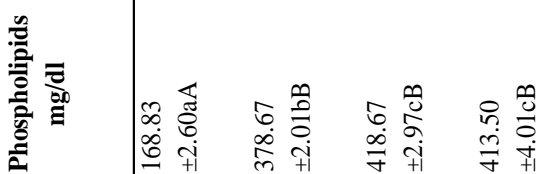

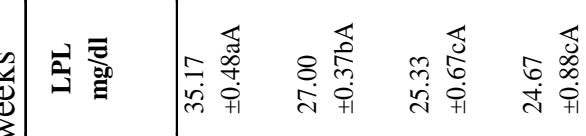

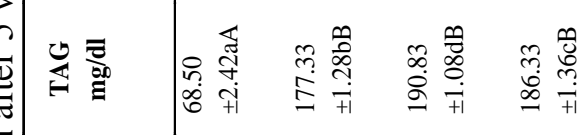

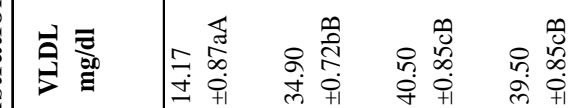

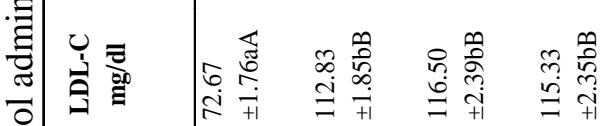

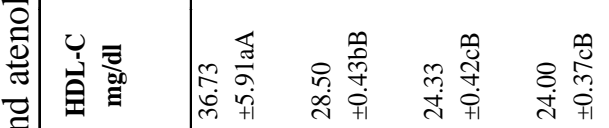

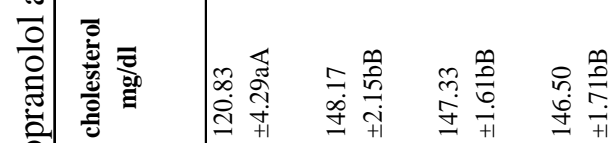

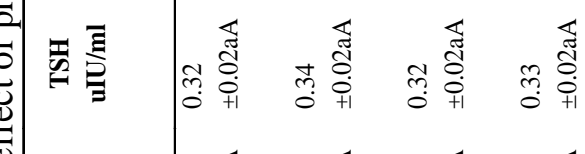

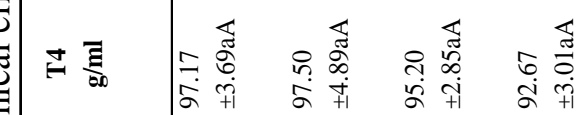

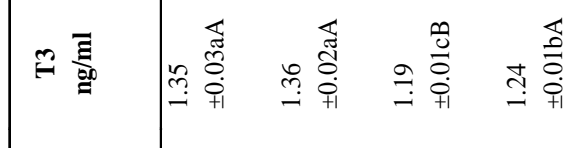

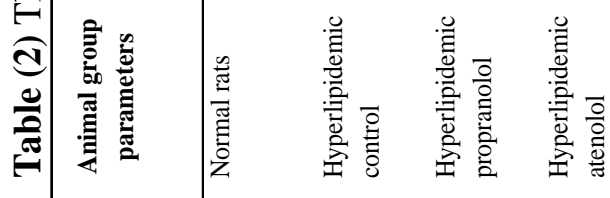




\section{References}

[1]Y.Ahmed, B.Dallal, K.Rawaa, M.K.Jaber, A.Hamo, Measurement of lipid profile parameters in hypertensive patients using atenolol or captopril; Ann. Coll. Med. Mosul; vol.36(1, 2), pp.41-48, 2011.

[2] J.D.Bauer, Clinical Laboratory Methods, the C.V. Company Waistline Industrial Missouri, 9th Ed, Chapter 33, P.555, 1982.

[3] H.K.Chopra, C.K.Krishna, S.S.Ravinder, K.K.Komal. Non-cardiac effects of atenolol. Supplement of JAPI, vol.57, pp.26-8, 2009.

[4] H.Drexel, F.W.Amann, F.Follath, H.R.Schmid, Effects of bisoprolol on lipoprotein cholesterol subfractions and apolipoproteins in patients with hypertension; J. Clin. Basic Cardiol.; vol.4(1), pp.57-60, 2001.

[5] R.Dhingra, P.Gona, E.J.Benjamin, T.J.Wang, J.Aragam, Relations of serum phosphorus levels to echocardiographic left ventricular mass and incidence of heart failure in the community. Eur. J. Heart Fail. Vol.12, pp.812-818, 2010.

[6] Y.Erbil., M.Giris, A.Salmoshoglu, Y.Ozluk, U.Barbaros, B.T.Yanuk, The effect of antithyroid drug treatment duration on thyroid gland micro vessel density and intraoperative blood loss in patients with Graves' disease; vol.143(2), pp.216-25, 2008.

[7] W.T.Friedewald, R.J.Levy, D.S.Fredrickson, Estimation of the concentration of LDL-c in plasma without use of preparative ultracentrifuge. Clin. Chem.; vol.18, pp.499502, 1972.

[8] P.Fossti, L.Prencipe, Serum Triglycerides determination colorimetrically with an enzyme that produces hydrogen peroxides. Clin. Chem., vol.28, pp.2077-88, 1982.

[9] R. J.Gibbons, Circulation. Am. Heart Ass. J. vol.106, p.3332, 2002.

[10] H.Gindler, D.king, Rapid colorimetric determination of calcium in biological fluids with methylene blue. Am. J. clin. Path. vol.58, pp.376-382, 1972.

[11] C.Harvengt, F.R.Heller, P.Martial, Y.Van, Short-term effects of beta-blockers atenolol, nadolol, pindolol, propranolol on lipoprotein metabolism in normolipidemic subjects. J. Clin. Pharmacol, vol.17, pp.475- 7, 2007.

[12] E.Huang, W.Kuo, Y.Chen, Homocysteine and other biochemical parameters in type 2 diabetes mellitus with different diabetic duration or diabetic retinopathy. Clin. Himica. Acta., vol.366, pp.293-8, 2006.

[13] A.A.Inaam, The Effect of Atenolol on CK-MB Levels in Hypertensive Patients. Iraqi J. Pharm. Sci. vol.18(1), pp.511-517, 2009.

[14] Jansen, Hans, Baggen, G.A.Rini, Effects of Doxazosin and Propranolol Administration on Lipoprotein Lipases in Cholesterol-Fed Rats. J.
Cardiovas. Pharmacol, vol.11, pp.303-307, 1987.

[15] W.Junge, K.Levabold, B.Kraak, Clinical Laboratory Methods. J. Clin. Chem. Clin. Biochem., vol.21, pp.445-451, 1983.

[16] A.W.Kung, "Clinical review: Thyrotoxic periodic paralysis: a diagnostic challenge". J. Clin. Endocrinol. Metab. Vol.91(7), pp.2490-5, 2006.

[17] V.Lopez, P.Stone, S.Ellis, J.A.Cowell, Cholesterol determination in HDL separated by three different methods. Clin. Chem. Vol.23(5), pp.882-4, 1977.

[18] M.Mahmoud, J.K.Mahmoud, K.Mohamed, A.Elham, Study on the effect of Paliurus spinachriti on Cholesterol, triglyceride and HDL levels in diabetic male rats fed a high cholesterol diet. Iran. J. of pharm. vol.3, pp.5154, 2004.

[19] M.Mazin, Shikara, M.Rajen, S.William, R.Seema, G.Jonas, The Effect of Propranolol, Atenolol and Metoprolol on the Serum Levels of Thyroxin (T4) and Triiodothyronine (T3). Proc. West. Pharmacol. Soc. Vol.46, p.127, 2003.

[20] S.M.McCann, Lipopolysaccharide-induced leptin release is neurally controlled. The Nat. Acad. Sci. vol.19, pp.167-171, 2001.

[21] L.R. McDougald, , G.F.Mathis, B.P. Seibert, Anticoccidial efficacy of diclazuril against recent field isolates of Eimeria from commercial poultry farms. Avi. Dis., vol.34(4), pp.911-915, 1990.

[22] K.N.Naito, A.Kaplan, Lipids Clin. Chem. The C.V. Mosby Co. St. Louis. Toronto Princeton; 918-919 and 570-572, 1984.

[23] E.Pasini, C.Opasich, R.Aquilani, Catecholamines and beta blockers in heart failure: Ital. Heart J. vol.1(2), p.171, 2000.

[24] P.Pothiwala, S.N.Levine, Analytic review: thyrotoxic periodic paralysis: a review". J. Intensive Care Med. vol.25(2), pp.71-7, 2010.

[25] R.A.Pulla, M.A.Syed, A.Mohammed, Effect of aged garlic extract and s-allyl cysteine and their interaction with atenolol during isoproterenol induced myocardial toxicity in rats. Indian $\mathbf{J}$. Pharmacol. Vol.46(1), pp.94-99, 2014.

[26] K.Samin, M.D.Sharma, Annapoorna, M.D.Kini, MRCP, D.Jonathan, M.D.Marmur, M.D.Valentin, P.D. Cardioprotective Effect of Prior Beta-blocker Therapy in Reducing Creatine Kinase-MB Elevation after Coronary Intervention, Circulation. Vol.102, pp.166-172, 2000.

[27] Scientific Committee, Recommendation pour la measure de la concentration de lactate deshidrogenasedans le serum human a $30^{\circ} \mathrm{C}$. Ann. Biol. Clin., vol.40, pp.87-164, 1982. 
[28] M.Somnath, D.Saibal, B.Sanjib, D.Ankur, S.Souvik, Pradip, G.Kumar, K.T.Santanu, Hepatotoxicity of atenolol therapy - A report of 2 cases. vol.189(13), pp.37-47, 2013.

[29] M.Soos, K.Siddle, Biochemical tests. J. Immunol. Methods, vol.51, pp.57-68, 1982.

[30] R.Steel, J.Torrie, D.Dickey, Principles and procedures of Statistics: A Biometrical Approach, McGraw-Hill, New York, NY, 3rd ed., 1997.

[31] M.Tabassum, Salahudin. Blood pressure lowering effects of atenolol in rats: Role of NaK-ATPase, serum, red cell and tissue electrolytes, Pakist. J. Pharmacol. Vol.19(2), pp.13-19, 2002.

[32] S.Tay, E.Khoo, C.Tancharoen, I.Lee, Betablockers and the thyrotoxic patient for thyroid and non-thyroid surgery: a clinical review. OA Anaesthetic. Vol.1(1), p.5, 2013.

[33] P.Urdal, S.Landaa, Macro creatine kinase BB in serum and some data on its prevalence. Clin. Chem, vol.25, pp.461-465, 1979.
[34] N.K.Virdi, D.J.Worthington, Cholesterol and Triglyceride stability in whole blood Ann.Clin .Biochem. vol.26, pp.197-8, 1989.

[35] W.H.C.Walker, Introduction: An Approach to Immunoassay. Clin. Chem. Vol.23, p.384, 1977.

[36] G.B.Wistom, Enzyme-Immunoassay. Clin. Chem. Vol.22, p.1243, 1976.

[37]Z.Xingguang, L.Xiaofeng, Z.Yanqi. J.Xiumin, Bin, Chen. Propranolol Prevents Osteoporosis and up-regulates Leptin in Ovariectomized Rats. Iran J Pharm Res. vol.12(3), pp.557-562, 2013.

[38] T.Yorio, G.DeLoach, N.Satumtira, Effects of antiglaucoma drugs on [32P] orthophosphate incorporation into phospholipids of cat iris and ciliary process. J Ocul Pharmacol. Fall; vol.1(3), pp.245-54, 2001.

[39] K.Young, Clin. Chem. Vol.21(1), p.D432, 1975.

[40] D.S.Young, Effects of disease on clinical lab tests. Circulation, vol.32(11), pp.283-286, 2001 

